\title{
La multidisciplina en los estudios universitarios. La perspectiva de los alumnos de la UAM-Iztapalapa
}

\author{
Juan-Manuel Hernández-Vázquez, Marco-Antonio Leyva-Piña y Javier Rodríguez-Laguna
}

\section{RESUMEN}

Este artículo pretende aportar elementos para valorar, desde la perspectiva estudiantil, la pertinencia de la reforma al modelo de formación universitaria, en lo tocante al enfoque multidisciplinar. La gran mayoría de las universidades públicas mexicanas han realizado ajustes curriculares en este sentido, mediante la incorporación vehemente de asignaturas pertenecientes a múltiples disciplinas. El campus Iztapalapa de la Universidad Autónoma Metropolitana (UAM) ha procedido así en la mayoría de los planes de licenciatura de sus tres divisiones académicas. No obstante, a 17 años de haberse iniciado el proceso de incrustación de cursos optativos multidisciplinares, aún no se sabe cuál ha sido el resultado de esta política. El estudio presentado aquí abona a subsanar dicha deficiencia, apelando a las opiniones de sus alumnos sobre la utilidad de la información y la orientación institucional recibidas, sus motivaciones y el cumplimiento de sus expectativas, la pertinencia de dichos cursos, y sus propuestas para mejorarlas.

Palabras clave: multidisciplinariedad, reforma curricular, currículo flexible, opiniones de los estudiantes, UAM, México.

\section{Juan-Manuel Hernández-Vázquez}

jm.uami@gmail.com

Mexicano. Doctor en Estudios Sociales por la Universidad Autónoma Metropolitana-Iztapalapa (UAM-1), México. Profesor investigador y coordinador del programa de Maestría y Doctorado en Estudios Sociales-Línea Estudios Laborales, UAM-I. Miembro del SNI, nivel 1. Temas de investigación: reforma universitaria, educación y mercados de trabajo, habitabilidad educativa de los espacios escolares. investigador del Departamento de Sociología, UAM-I. Temas de investigación: reforma universitaria, modelos universitarios, relaciones laborales. 


\title{
A multidisciplina nos estudos universitários. A perspectiva dos alunos da UAM-Iztapalapa
}

\section{RESUMO}

Este artigo pretende fornecer elementos para avaliar, segundo a perspectiva estudantil, a pertinência da reforma ao modelo de formação universitária, no tocante ao enfoque multidisciplinar. A grande maioria das universidades públicas mexicanas têm realizado ajustes curriculares neste sentido, mediante a incorporação veemente de matérias pertencentes a múltiplas disciplinas. O campus Iztapalapa da Universidade Autônoma Metropolitana (UAM) tem procedido assim na maioria dos planos de licenciatura de suas três divisões acadêmicas. Não obstante, a 17 anos de haver iniciado o processo de incrustação de cursos optativos multidisciplinares, ainda não se sabe qual foi o resultado desta política. O estudo apresentado aqui ajuda a corrigir dita deficiência, apelando às opiniões de seus alunos sobre a utilidade da informação e a orientação institucional recebidas, suas motivações e o cumprimento de suas expectativas, a pertinência de ditos cursos, e suas propostas para melhorá-las.

Palavras chave: multidisciplinariedade, reforma curricular, currículo flexível, opiniões dos estudantes, UAM, México.

Multidisciplinary approaches in university studies. The perspective of the students enrolled in the UAM-Iztapalapa

\begin{abstract}
This article aims to provide elements to assess, from the students' perspective, the relevance of the reform to the university training model, in terms of the multidisciplinary approach. The great majority of mexican public universities have made curricular adjustments in this sense, through the emphatic incorporation of subjects belonging to multiple disciplines. The Iztapalapa campus of the Metropolitan Autonomous University (Universidad Autónoma Metropolitana, UAM) has done so in most of the degree plans of its three academic divisions. However, 17 years after the process of inserting multidisciplinary elective courses began, the result of this policy have not been showed yet; this study contributes to repair this lack, appealing to the opinions of its students on the usefulness of the information and institutional orientation received, their motivations and the fulfillment of their expectations, the relevance of these courses, and their proposals for improvement.
\end{abstract}

Key words: multidisciplinary approach, curriculum reform, flexible curriculum, student opinions, UAM, Mexico. 


\section{Introducción. La reforma curricular en la UAM-Iztapalapa}

A finales de los años 1990, en la Universidad Autónoma Metropolitana (UAM) dio inició un proceso de reflexión autocrítica sobre la docencia, del cual derivó el documento de diagnóstico Reflexión de la Universidad desde la Docencia (UAM, 2001). En él se destacaba la preocupación porque el sistema de becas y estímulos al personal académico, implementado desde 1989, había contribuido al debilitamiento no sólo de la práctica docente en sí, sino también del vínculo docencia-investigación, mismo que había constituido uno de los pilares centrales del modelo de organización departamental con que había nacido la institución en 1974. Dicho proceso culminó en 2001 (UAM, 2001) con la aprobación de una reforma que la Rectoría General instituyó mediante las Políticas Operacionales de Docencia (POD) (UAM, 2018), las cuales guiaron a las unidades académicas para lanzar sus propias Políticas Operativas de Docencia (PODI, en el campus Iztapalapa). En 2003, las PODI (UAM-I, 2003) decantaron las propuestas específicas de los académicos de la UAM-Iztapalapa para mejorar su práctica docente, haciendo descansar, en varias políticas, el objetivo de ofrecer una formación integral, entre ellas la encaminada a flexibilizar los planes y programas de estudio, mediante la incorporación de asignaturas multidisciplinarias.

Han pasado ya 17 años desde el lanzamiento de las PODI, pero aún no es claro el resultado alcanzado, tanto en cada división académica, como en el conjunto de la Unidad Iztapalapa. De hecho, hasta el momento no existe un plan para evaluar los resultados de esas políticas, en particular la de flexibilidad curricular. En cada plan de estudios reformado se decantaron propuestas, cuyos impactos en las prácticas docentes y en el aprendizaje de los alumnos esperan aún ser analizados. No existe información institucional para valorar los resultados alcanzados por el modelo educativo basado en la flexibilidad curricular y las demás políticas operativas de docencia.
Los informes oficiales de cada rector de la Unidad muestran resultados en el número de currículos ajustados, pero no informan, por ejemplo, porque no se ha indagado cuál ha sido su impacto en los aprendizajes, en la inserción laboral de los egresados y en las prácticas docentes concretas.

En tanto que la flexibilidad curricular se ha afianzado como práctica recurrente en las universidades, es importante saber cuál es la perspectiva de los actores educativos sobre la misma, dado que su implementación encontró asidero en la doble expectativa de lograr una novedosa docencia, basada en la composición interdisciplinar de la plantilla académica, en combinación con la formación de un alumnado comprometido con su propio aprovechamiento, participativo y con autonomía académica.

Este artículo pretende aportar elementos para la mejora en los procesos de toma de decisiones, sustentadas sobre el diseño e implementación de políticas de flexibilización curricular. Para ello se transita por dos vías, en la primera se discuten las ideas más recurrentes que abogan por una enseñanza superior flexible, y en la segunda se examina la experiencia de los estudiantes de la UAM-I, al enfrentarse con un modelo curricular multidisciplinario diseñado bajo estas ideas.

Se aprovecha información levantada censalmente a mediados de 2014, sobre 460 estudiantes que en ese momento estaban cursando asignaturas optativas distintas a las contenidas en el currículo disciplinar troncal, generalmente impartidas en divisiones académicas distintas a la de adscripción, para analizar sus apreciaciones sobre la utilidad de la información y la orientación recibidas, las motivaciones que guiaron la elección de dichas asignaturas, la pertinencia de las mismas, el cumplimiento de sus expectativas, y sus propuestas de mejora.

\section{Flexibilidad curricular, ¿innovación pedagógica o presión del mercado?}

Desde el inicio del nuevo siglo han ocurrido cambios económicos, políticos, sociales, culturales y 
tecnológicos que han impactado fuertemente a la sociedad. En el campo educativo resaltan las presiones de organismos internacionales financieros y educativos para adaptar la educación a entornos económicos inestables, volátiles, rentables y con aumentos en los niveles de consumo (Maldonado, 2000).

En la definición de las reformas universitarias nacionales destaca la influencia de las recomendaciones que diversos organismos internacionales han manifestado a través de varios documentos, como la Declaración Mundial sobre la Educación Superior en el Siglo XXI de la UNESCO (1998), las declaraciones que hicieron en La Sorbona, Bolonia y Berlín los ministros europeos de educación superior $(1998,1999$ y 2003), y las conclusiones del Consejo Europeo (2002) en Barcelona. En cada país, esas encomiendas han sido dotadas de significados propios. En América Latina han jugado el papel de presiones externas para adaptar la educación a las exigencias del mercado. Así, han influido en la definición de acciones conjuntas de gobierno y empresarios, dirigidas a orientar el campo educativo en un proceso creciente de mercantilización de la educación (García, 2010).

Las reformas educativas a nivel mundial comparten una serie de expectativas y de formas de acción que buscan, a través del cambio, la conformación de nuevos actores educativos: profesores y alumnos asumiendo el mercado, adaptándose a las demandas de las flexibilidades laboral y educativa (SEP, 2016).

En este escenario de transformación educativa se ha impuesto un discurso que, con su propio lenguaje prescriptor, impulsa la construcción de una nueva normalidad en las prácticas educativas de profesores y alumnos, bajo las ideas de corresponsabilidad, participación y autonomía en los aprendizajes. En lo formal, estas ideas parecen adecuadas para su incorporación por parte de los actores educativos, sin embargo, en lo concreto deben ser valoradas en cuanto a su función como impulsoras de la mercantilización de la educación (Suárez, 2011), y en cuanto a la posibilidad real de que los actores educativos asuman un papel activo en los procesos implicados. Este papel es de difícil construcción si en lugar de ser algo conscientemente buscado en la implementación de procesos innovadores de enseñanza-aprendizaje, lo que en realidad se hace es inocular prácticas educativas forzadas, sin las necesarias mediaciones sociales de quienes serán sus realizadores (Díaz Barriga F., 2005; De la Peña, 2007; Gil, 2012), mismos que en el día a día podrían generar las opciones pedagógicas adecuadas, en tanto partícipes directos en los procesos, o limitar los efectos de la gestión escolar, dado el control, que en los hechos tienen del aula y de los elementos activos en ella (Montes et al., 2017).

Entre las prescripciones del nuevo discurso resaltan algunas reconocidas como innovaciones educativas (Cano, 2009): modelos de competencias como estrategias determinantes del aprendizaje; metodologías didácticas para la creación de interacciones dialógicas en el aula; tutorías para los alumnos; diseño de perfiles de egreso orientados a las exigencias de los mercados de trabajo; planes de estudio y programas de asignaturas abiertos a la interdisciplina y a la multidisciplina; certificaciones externas a las universidades; atracción del sector privado para patrocinar proyectos de investigación; incentivos económicos para motivar y arraigar al personal docente, ligados a evaluaciones cuantitativas del desempeño. Pero éstas parecen recetas complejas que no consideran detenidamente el tipo de vínculo que debería darse entre educación, economía y mercado, en términos de su pertinencia, no sólo económica, sino también pedagógica y educativa integral (Torres, 1998; Hargreaves y Fink, 2006; Díaz Barriga A., 2005; Escudero, 2006).

Cabe reflexionar, además que como los procesos de reforma en las instituciones de educación superior (IES) necesariamente ocurren en el terreno de las experiencias comunicativas, y de la interiorización de los conceptos involucrados en ellas (Ziegler, 2003), entonces el dilema crece si se intenta la imposición de tales procesos como normas de autoridad. Para 
que la flexibilidad curricular se convierta en una estrategia con impacto transformador de la práctica educativa en el aula, es importante que profesores y alumnos sean tomados en cuenta como sujetos activos, con capacidad para incidir positivamente en los procesos de enseñanza-aprendizaje. Los primeros como diseñadores y operadores de innovaciones curriculares en el aula, y los segundos como sujetos con capacidad para proponer mejoras, no como meros entes receptores y repetidores de prescripciones definidas por las autoridades educativas.

A las preocupaciones por los magros resultados obtenidos con la implementación de la flexibilidad curricular en las IES (Caamal y Canto, 2009; Martínez et al., 2009), se le suma otra relacionada con la implementación de la idea de formar alumnos activos en la toma de decisiones sobre la planificación de su propio currículo, porque en los hechos, frecuentemente el cumplimiento de esta prescripción se reduce a concederles la posibilidad de elegir sus materias optativas, ya sean éstas del mismo tronco disciplinar de la carrera que cursan, o de campos multidisciplinares.

La cuestión de tener un modelo educativo centrado en el alumno, suele asumirse en las IES sin ir más allá de darle oportunidad para que tome decisiones sobre la organización de su historia académica, lo que constituye una autonomía formalista y limitada, que dista mucho de llegar hasta el aula, espacio que frecuentemente continúa siendo el imperio de profesores con tendencia a organizar la enseñanzaaprendizaje tal como ellos la vivieron cuando fueron alumnos.

Esta concepción limitada de la autonomía estudiantil presenta problemas sensibles en sus formas de operación, porque las elecciones sobre las asignaturas optativas, en una estructura de currículo con tintes multidisciplinares, frecuentemente implican significativos problemas comunicativos y de sentido para la formación universitaria, puesto que lejos de ser resultado de razonamientos académicos, suelen descansar en razones pragmáticas, como cuando las elecciones se basan, no en el interés por aprender los contenidos, sino en la facilidad para concluir la carrera, escogiendo determinados horarios y grupos con amplios cupos, sin importar la materia.

Amén de lo anterior, una cuestión toral para entender la complejidad de la docencia en la actualidad, que va más allá de cuestiones técnicas y operativas, es la proliferación y diversidad de prescripciones impulsadas desde la política educativa a nivel internacional sobre la interdisciplina y la multidisciplina, mismas que se presentan como las opciones de futuro en el aprendizaje de los alumnos, dadas las nuevas orientaciones académicas derivadas del nuevo paradigma científico (Morin, 1998).

Es necesario considerar que este modelo prescriptor se enfrenta a una serie de controversias centradas en la ambigüedad conceptual de lo que se entiende por interdisciplina, multidisciplina, transdisciplina y neodisciplina. Sobresalen algunas ideas sobre estas formas de modelación curricular, como las siguientes: es la única forma de hacer ciencia en la actualidad; la investigación se hace de mejor forma cuando se juntan dos o más especialidades científicas; juntar lo que está separado, tomar un conocimiento de aquí y otro de allá, permite el descubrimiento de nuevo conocimiento; las disciplinas se encuentran limitadas para la atención de temas frontera; es una estrategia para vincular teorías diferentes, a fin de tratar problemas complejos más eficientemente; es la participación de varias disciplinas; es la reunión de diferentes enfoques para enriquecer la mirada respecto a un problema concreto; provoca creatividad en los alumnos; siembra clases participativas; promueve el trabajo en equipo; prepara para el trabajo moderno; forma al alumno para la articulación de conocimientos diversos; resuelve el problema de la docencia, y finalmente, se dice también que implica una cuestión de actitud científica. Incluso se ha pensado, de manera extremista, que la exigencia moderna del fin de las disciplinas y la emergencia de un nuevo paradigma 
científico implica la unificación de la ciencia (Salvador, 2014; Kravzov, 2000; Santoyo, 1996).

Cabe comentar que la complejidad polisémica de estas ideas parece ser resultado de su uso pragmático y poco razonado a la hora de diseñar los programas de estudio, lo cual da origen a un complejo conjunto de tensiones que, en la práctica, han de ser gestionadas directamente por los actores educativos. Se soslaya que la aplicación de estas orientaciones, en el ámbito de la enseñanza-aprendizaje, no necesariamente tiene la misma naturaleza y forma que en el campo de la investigación.

En la literatura especializada se reconocen diferentes orígenes de esas estrategias de conocimiento, y diferentes formas de aplicarlas a la docencia, así como diferentes momentos de desarrollo y opacidad (Follari, 2007). Sin embargo, en la actualidad se apela constantemente a estas orientaciones formativas como instrumento propiciador de aprendizajes enriquecidos. En la Red Innova Cesal (REDIC), se han preguntado qué se entiende y cómo debe abordarse la interdisciplinariedad en las currículas universitarias, para no sumarse acríticamente a la alucinante fascinación por la misma (Quintá, 2014). Es necesario reparar en la naturalización que se ha hecho de esta cuestión, a fin de evitar el diseño de planes de estudio en los que, al final, las asignaturas pertenecientes a distintas disciplinas quedan vinculadas forzadamente. La experiencia indica que aún está pendiente la búsqueda de modalidades de organización curricular colegiada, mediante la cual los participantes, en un diálogo multidisciplinar, encuentren una forma eficaz para transformar las prácticas docentes ancladas en los enfoques disciplinares.

De acuerdo con diferentes autores, como los participantes en la REDIC, el auge de la interdisciplinariedad requiere reflexión teórica y una adecuada problematización práctica, para implementarla en el aula. Esta sugerencia implica la necesidad de que la organización curricular repase las formas eficaces de vincular asignaturas disciplinares y multidisciplinares, y la manera de combinarlas con estrategias para la construcción de ambientes de aprendizaje pensados para los alumnos.

Además, siguiendo el razonamiento de Follari (2007), la interdisciplinariedad aspira a conformar diseños curriculares que presuponen trabajo colegiado, e implica la creación de condiciones académicas y administrativas flexibles, así como la participación de una planta docente activada en el convencimiento de las bondades inherentes a la comunicación entre disciplinas.

No se puede obviar el hecho de que el trabajo docente interdisciplinar implica prácticas y culturas docentes específicas, para afrontar responsabilidades laborales adicionales a las tradicionales, como la planeación conjunta de las actividades que han de realizarse en el aula; el manejo de los criterios de aprendizaje, valores y actitudes del modelo de enseñanza centrado en el alumno, y de manera especial, la realización de labores de seguimiento a la estrategia de interdisciplinariedad aplicada, para valorar el cumplimiento de los objetivos.

Sin desconocer el florecimiento cognitivo que representa la interdisciplina y la multidisciplina para el aprendizaje, pocas investigaciones han podido dar cuenta del cumplimiento en las aulas universitarias de las promesas antes expuestas. Es necesaria la sistematización y difusión de experiencias de docencia para asegurar su éxito operativo, en un mundo académico en el que destacan las deducciones teóricas y las prescripciones (Spelt et al., 2009).

\section{Metodología}

Las Políticas Operativas de la Docencia en la UAM han orientado desde 2003 las transformaciones curriculares en las licenciaturas de sus tres divisiones académicas (Ciencias Básicas e Ingeniería, CBI; Ciencias Sociales y Humanidades, CSH; y Ciencias Biológicas y de la Salud, CBS), con la pretensión de formar integralmente al estudiante bajo cuatro políticas orientadoras: flexibilidad curricular, 
aprendizaje autónomo corresponsable con la labor de los profesores, desarrollo de habilidades básicas, y revitalización del añorado vínculo entre docencia e investigación (UAM-I, 2003).

La implementación de la flexibilidad curricular ha implicado la inclusión de tres núcleos de multidisciplina obligada en los planes de estudio (todos duran 12 trimestres). El primero tiene lugar durante la primera mitad de la carrera correspondiente, en el tronco general divisional, e incluye asignaturas no elegibles, propias de varias disciplinas pertenecientes a la división académica de adscripción. El segundo se da en la segunda mitad, y requiere que sean cursadas asignaturas ofrecidas también por la misma división, cuya elección se deja al juicio del alumno, siempre y cuando estén incluidas en un listado predefinido por el Colegio de Profesores de la carrera correspondiente. Estas asignaturas son conocidas en la UAM-I como "Optativas Divisionales". Finalmente, el tercer núcleo de multidisciplina también ocurre en la segunda mitad de la carrera, y también involucra optativas multidisciplinarias, pero diseñadas y gestionadas por otras divisiones académicas, por eso son llamadas "Optativas Extradivisionales" (OEX). Esta clase de asignaturas multidisciplinarias fueron las enfocadas por el estudio aquí reportado.

Para avanzar en desentrañar las apreciaciones de los alumnos de la UAM-I sobre las asignaturas OEX, se diseñó un levantamiento censal exploratorio con fines fundamentalmente descriptivos, que abarcó a todos aquellos que al final del trimestre de primavera 2014, se encontraban activos en esta clase de asignaturas, independientemente de la división de adscripción. Así, se aplicó el instrumento La multidisciplina desde la experiencia de los alumnos UAM-I 2014, entrevistando a 460 alumnos, cifra que representó aproximadamente al 90\% de los alumnos con actividad en las OEX al final de dicho trimestre (Hernández y Leyva, 2015). En el nivel de las divisiones académicas, en CBI se entrevistaron 27 de los 28 alumnos integrantes de la población objetivo; en CBS, 59 de
62, y en CSH, 374 de 430. Participaron todas las carreras ofrecidas en el campus, salvo las que al final del trimestre no tenían alumnos activos en las OEX: Ingeniería Biomédica y Computación, de CBI; Ciencia Política, Geografía Humana y Psicología Social, de CSH, e Ingeniería de los Alimentos, de CBS.

Para emprender una aproximación a entender el papel jugado por la UAM-I en la implementación de las asignaturas OEX y su importancia curricular, desde la perspectiva de los alumnos, fueron construidos tres índices sumativos simples: Utilidad de la Información Institucional (UTIN) sobre esas asignaturas; Utilidad de la Orientación Institucional (UTOR) sobre las mismas; y Pertinencia (PERT) de las mismas. El instrumento y más detalles metodológicos pueden ser consultados en Hernández y Leyva (2015). Cabe aclarar que la construcción de estos índices incluyó solamente las variables que saturaron satisfactoriamente en cada uno de ellos. El proceso para fijar las variables constitutivas siguió la técnica de Análisis Factorial por Componentes Principales, descartando en cada iteración las variables con escasa saturación, hasta que en cada caso la medida de adecuación muestral Kaiser-Meyer-Olkin (KMO) alcanzó valores mayores a 0.5, y la medida Alfa Ordinal, de confiabilidad de escala, registró valores por arriba de 0.8.

\section{Resultados}

Con el apoyo de los tres índices sintéticos antes mencionados, y otra información relevante levantada también en el ejercicio censal señalado, en este apartado se indagan varias cuestiones sobre las OEX: primero se busca saber cuál es la utilidad de las fuentes institucionales y no institucionales de información y orientación, a las que los estudiantes recurren para tomar decisiones sobre cuáles de estas asignaturas conviene cursar; luego se averigua qué razones tienen para elegirlas, y qué tanto se cumplen sus expectativas al cursarlas; posteriormente se analiza qué piensan sobre la pertinencia de las mismas para su formación profesional, y finalmente, se 
exponen las propuestas de los propios estudiantes para mejorarlas.

\section{Información para elegir las multidisciplinarias}

Partiendo del supuesto de la constitución de alumnos con autonomía para diseñar su formación académica, nos preguntamos acerca de cuáles son sus apreciaciones respecto a las OEX cursadas, en cuanto a la información y orientación que recibieron para cursarlas y a las experiencias subjetivas derivadas de las interacciones profesor-alumno y alumno-alumno.

El ejercicio censal realizado arrojó que casi todos los alumnos $(97.6 \%)$ recibieron algún tipo de información previa a la elección de esas asignaturas, y de ellos, un $78.2 \%$ la obtuvieron de alguna instancia de la UAM-I. El ejercicio encontró que la información es ponderada con más utilidad positiva que negativa (cuadro 1).
Además, se encontró que dicha información tiene más utilidad para los estudiantes si fue recibida de profesores o compañeros, antes que de la fuente propiamente institucional, aunque no quita que esos mismos informantes en un momento anterior la hubieran conseguido por los canales institucionales.

$\mathrm{Al}$ parecer, la utilidad es más frecuentemente señalada por los hombres que por las mujeres, y básicamente consideran que la fuente más útil proviene de sus compañeros (cuadro 2). Es claro que las conversaciones entre ellos tocan información sobre diversos aspectos de las OEX, de quiénes las imparten y sus capacidades de docencia, inclusive de lo fácil o dificil que sería obtener una nota aprobatoria o excelente. Muchas de éstas son informaciones difícilmente obtenibles por otros medios, y que no suelen estar al alcance de los recursos institucionales.

Cuadro 1. Recepción de información antes de elegir la multidisciplinaria y su utilidad, según la fuente (\%)

\begin{tabular}{|c|c|c|c|c|}
\hline Fuente de información & Dio inf. & Inútil & Útil & Total \\
\hline Sistemas escolares & 50.9 & 44.7 & 55.4 & 100 \\
\hline Coordinador de tu carrera & 58.3 & 43.8 & 56.2 & 100 \\
\hline Autoridades universitarias & 45.4 & 58.2 & 41.8 & 100 \\
\hline Profesores & 64.0 & 29.7 & 70.3 & 100 \\
\hline Informació & entaria & & & \\
\hline Mis compañeros de la UAM-I & 89.7 & 13.8 & 86.2 & 100 \\
\hline Otra persona ajena a la UAM-I & 18.5 & 46.8 & 53.2 & 100 \\
\hline Recibió información de al menos una de las fuentes & \multicolumn{4}{|c|}{97.6} \\
\hline Recibió información de al menos una fuente UAM-I & \multicolumn{4}{|c|}{78.2} \\
\hline
\end{tabular}

Fuente: cálculos propios con base en el censo La multidisciplina desde la experiencia de los alumnOS, UAM-I 2014. 
Cuadro 2. Porcentaje de información útil, según su procedencia, sexo y división

\begin{tabular}{l|c|c|c|c|c|c|c}
\hline \multicolumn{1}{c|}{ Fuente de información } & H & M & CBI & CSH & CBS & Total \\
\hline Sistemas escolares & 61.8 & 49.6 & 70.6 & 51.9 & 69.0 & 55.4 \\
\hline Coordinador de carrera & 57.6 & 55.0 & 63.2 & 55.6 & 55.9 & 56.2 \\
\hline Autoridades universitarias & 47.4 & 36.9 & 50.0 & 40.2 & 48.1 & 41.8 \\
\hline Profesores & 72.6 & 68.6 & 66.7 & 69.0 & 79.1 & 70.3 \\
\hline
\end{tabular}

H: Hombre; M: Mujer; CBI: Ciencias Básicas e Ingeniería; CSH: Ciencias Sociales y Humanidades; CBS: Ciencias Biológicas y de la Salud.

Fuente: cálculos propios con base en el censo La multidisciplina desde la experiencia de los alumnos, UAM-I 2014.

Cuadro 3. Media y mediana del índice UTIN, según sexo y división

\begin{tabular}{|c|c|c|c|c|c|c|c|c|c|c|c|c|}
\hline \multirow{2}{*}{ Valor a mitad de escala UTIN } & \multicolumn{6}{|c|}{ Media } & \multicolumn{6}{|c|}{ Mediana } \\
\hline & $\boldsymbol{H}$ & $\boldsymbol{M}$ & $C B I$ & $\mathrm{CSH}$ & CBS & Total & H & $M$ & $C B I$ & $\mathrm{CSH}$ & CBS & Total \\
\hline 10 & 11.0 & 10.1 & 11.6 & 10.4 & 11.3 & 10.6 & 11.0 & 10.0 & 11.0 & 10.0 & 12.0 & 11.0 \\
\hline
\end{tabular}

UTIN: Utilidad de la información sobre las multidisciplinarias; H: Hombre; M: Mujer; CBI: Ciencias Básicas e Ingeniería; CSH: Ciencias Sociales y Humanidades; CBS: Ciencias Biológicas y de la Salud.

Fuente: cálculos propios con base en el censo La multidisciplina desde la experiencia de los alumnos, UAM-I 2014.

En el nivel de las divisiones, los patrones de opinión manifiestan algunas variantes interesantes. En todas las divisiones, la fuente informativa más útil estuvo en los compañeros de estudio, y las menos útiles en autoridades universitarias y personas ajenas a la institución. Las diferencias se encontraron en el orden de utilidad concedido a las demás fuentes informativas: los profesores ocuparon el segundo lugar en CSH y CBS, pero el tercero en CBI; y los coordinadores, el tercero en CSH, pero el cuarto en CBI y CBS. Entonces, es posible establecer que la institución es percibida con fallas administrativas que cuestionan su apoyo para hacer de las OEX asignaturas con potencial académico. El índice UTIN (cuadro 3) ofrece valores que nos llevan a suponer que los alumnos se encuentran en el limbo de las percepciones hacia la institución; para ellos ésta no hace bien su trabajo, pero tampoco lo hace mal.

Posiblemente también se trate de la profundidad de la información que las coordinaciones de licenciatura y sus académicos deben manejar sobre este tipo de cursos, los cuales, dado que son de nivel superior, siempre deberían aportar algo que el futuro profesionista pudiera utilizar en el ejercicio de su profesión. De hecho, se requiere que la institución ofrezca información sobre las asignaturas OEX, de modo que los alumnos puedan dirimir si les serán no sólo útiles, sino también interesantes. Esto nos lleva a pensar en la ausencia de otro nivel de información que no opera en las interacciones "de pasillo" alumno-alumno o alumno-profesor, ni en la interacción "de mostrador" alumno-burocracia institucional. Si 
se desarrollara este nivel, cabrían mejores resultados en términos del impacto que traería para la vida profesional de los alumnos el cursar estas materias.

\section{Orientación académica}

Si se pensara, como parece ser el caso, que las OEX pueden abonar a la formación integral de los profesionistas en una época como la nuestra, posiblemente se tendría en mente que su relevancia es tal y no un mero formalismo. Por tanto, habría que tener la habilidad institucional para acercar a los estudiantes de las distintas carreras a ese conocimiento. Ya hemos visto que la información institucional no parece útil a los ojos de los estudiantes, pero parece que tampoco ocurre en el campo de la orientación académica que ofrece para poder elegirlas. De hecho, resulta que la orientación que reciben y les es útil, procede más de los propios estudiantes (81.3\%). Además, la información da idea de que la gran mayoría de los alumnos de la UAM-I (91.3\%) recibió alguna clase de orientación, pero sólo poco más de la mitad (55.4\%) la recibió por parte de algún académico de la institución (cuadro 4).

Cuadro 4. Recepción de orientación antes de elegir la multidisciplinaria y su utilidad, según la fuente (\%)

\begin{tabular}{|c|c|c|c|c|}
\hline Fuente de orientación & Dio orient. & Inútil & Útil & Total \\
\hline Tutor & 28.6 & 43.1 & 56.9 & 100 \\
\hline Asesor & 30.5 & 38.8 & 61.2 & 100 \\
\hline Profesor & 52.3 & 32.4 & 67.6 & 100 \\
\hline Informació & plementaria & & & \\
\hline Mis compañeros de la UAM-I & 85.7 & 18.7 & 81.3 & 100 \\
\hline Otra persona ajena a la UAM-I & 13.8 & 39.0 & 61.0 & 100 \\
\hline Recibió orientación de al menos una de las fuentes & \multicolumn{4}{|c|}{91.3} \\
\hline Recibió orientación de al menos una fuente UAM-I & \multicolumn{4}{|c|}{55.4} \\
\hline
\end{tabular}

Fuente: cálculos propios con base en el censo La multidisciplina desde la experiencia de los alumnos, UAM-I 2014.

Entre las fuentes académicas de orientación institucional consideradas, los tutores quedaron a la zaga porque fue calificada por los alumnos como la más escasa y menos útil. Asunto de particular interés por cuanto que una parte toral de las reformas que la institución ha hecho a partir de las PODI, ha sido justamente promover que sus académicos adquieran la figura de tutor entre sus funciones, más allá de la figura del asesor, que al parecer se limita a la orientación para los trabajos terminales de la licenciatura (tesinas). Del total de alumnos, sólo 28.6\% dijeron que dicha figura académica les había dado información y que, cuando se las dio, había sido inútil en el $43.1 \%$ de los casos. Este resultado se verifica en otras investigaciones que han llegado a conclusiones parecidas, teniendo como objeto de estudio a la UAM-I y sus estudiantes (Hernández y Rodríguez, 2015). Incluso la frecuencia con que las personas ajenas a la universidad ofrecieron información útil a los alumnos fue superior a la de los tutores. Si bien los alumnos recibieron escasa orientación de personas ajenas a la universidad $(13.8 \%$, familiares o 
egresados de la UAM, principalmente), cuando la recibieron resultó útil $61 \%$ de las veces, más de cuatro puntos porcentuales arriba de lo reportado para los tutores.

Igual que en cuanto a utilidad informativa, es notorio que en general las alumnas tienden a valorar más estrictamente que los hombres la utilidad en la orientación proporcionada. En definitiva, la lógica de esto es que, en orientación, las figuras de tutor y asesor son siempre menos útiles que la figura del profesor como tal (cuadro 5). En cambio, cuando se trata de la orientación proporcionada por los propios compañeros, las mujeres perciben más utilidad que los hombres por más de 6 puntos porcentuales.

Cuadro 5. Porcentaje de orientación útil, según su procedencia, sexo y división

\begin{tabular}{l|c|c|c|c|c|c|c}
\hline \multicolumn{1}{r|}{ Fuente de orientación } & H & M & CBI & CSH & CBS & Total \\
\hline Tutor & 61.3 & 52.9 & 66.7 & 55.5 & 64.3 & 56.9 \\
\hline Asesor & 67.2 & 56.0 & 66.7 & 60.0 & 69.2 & 61.2 \\
\hline Profesor & 68.6 & 66.9 & 58.3 & 66.8 & 75.8 & 67.6 \\
\hline
\end{tabular}

H: Hombre; M: Mujer; CBI: Ciencias Básicas e Ingeniería; CSH: Ciencias Sociales y Humanidades; CBS: Ciencias Biológicas y de la Salud.

Fuente: cálculos propios con base en el censo La multidisciplina desde la experiencia de los alumnos, UAM-I 2014.

A nivel de las divisiones, los patrones de opinión manifiestan variantes interesantes (cuadro 6). En CSH los porcentajes de orientación útil son relativamente menores a los registrados por las otras divisiones, salvo en el caso de la orientación del profesor, en contraste con CBI. En CBS la orientación de los profesores es considerada como relativamente más útil. Por su parte, en CBI no son los profesores quienes más frecuentemente ofrecen orientación útil, sino los tutores y los asesores. También sorprende CBI porque al contrario de CSH y más de CBS, la orientación recibida por personas ajenas al campus universitario, como familiares y egresados, es considerada la más orientadora, incluso mejor que la de los propios compañeros.

Posiblemente en CBI los tutores obtuvieron las mejores evaluaciones, debido al particular modo de gestión aplicado en esa división sobre dicha actividad académica. En CBI, las tutorías son organizadas y supervisadas más estrictamente que en las otras divisiones y los que no cumplen suficientemente con las mismas, tienen más trabas para obtener mejores montos en la compensación salarial llamada Beca de Docencia.

Un recurso para apreciar sintéticamente las opiniones estudiantiles sobre la utilidad de la orientación recibida por parte de agentes institucionales privilegiados (profesores, asesores y tutores) es el índice sobre la Utilidad de la orientación (UTOR). De acuerdo con los valores de la media y mediana de este índice para el conjunto de la UAM-I (8 y 9 , respectivamente, cuadro 6), al parecer los alumnos están percibiendo que la institución necesita estrategias más eficaces de orientación académica, y ya que dichos valores se ubican muy cerca de la mitad de la escala (7.5), se puede hablar de un papel orientador un tanto mediocre de la institución. 
Cuadro 6. Media y mediana del índice UTOR

\begin{tabular}{|c|c|c|c|c|c|c|c|c|c|c|c|c|}
\hline \multirow{2}{*}{ Valor a mitad de escala UTOR } & \multicolumn{6}{|c|}{ Media } & \multicolumn{6}{|c|}{ Mediana } \\
\hline & $\boldsymbol{H}$ & M & CBI & CSH & CBS & Total & $H$ & M & CBI & CSH & CBS & Total \\
\hline 7.5 & 8.5 & 7.6 & 10.0 & 7.9 & 8.6 & 8.0 & 9 & 8 & 11 & 8 & 9 & 9 \\
\hline
\end{tabular}

UTOR: Utilidad de la orientación sobre las multidisciplinarias; H: Hombre; M: Mujer; CBI: Ciencias Básicas e Ingeniería; CSH: Ciencias Sociales y Humanidades; CBS: Ciencias Biológicas y de la Salud.

Fuente: cálculos propios con base en el censo La multidisciplina desde la experiencia de los alumnos, UAM-I 2014.

El personal académico constituye el agente institucional fundamental de orientación para que los alumnos tomen mejores decisiones sobre su formación académica. Si falla en su función, entonces se pueden adivinar escenarios preocupantes en cómo los alumnos están eligiendo las OEX, y en las repercusiones académicas de dichas elecciones.

\section{Razones de elección y cumplimiento de expectativas}

Antes quedó claro que el proceso informativo y orientador sobre las OEX, liderado por la institución y sus académicos, se encuentra muy limitado. Lo que sigue, nos lleva a entrever la existencia de una dinámica por efecto de la cual, cursar estas materias podría limitar el desarrollo del estudiante. Si congregáramos las razones que esgrimieron los alumnos para determinar qué OEX cursarían en dos tipos: académicas y no académicas, tendríamos que las primeras influyeron en $64.9 \%$ de las elecciones, aunque entre ellas, la mitad (32.7\%) se basó tan sólo en que el nombre de la asignatura parecía interesante (cuadro 7). Esto no sorprende, dado que como ya se mencionó, la principal fuente de información y de orientación fue la representada por los propios compañeros. Adicionalmente, se estima que los procesos administrativos constituyen un aspecto preocupante para la definición de las OEX a cursar, porque en ocasiones dificultan que los alumnos concluyan sus estudios en los tiempos previstos por los programas de licenciatura. En este sentido, es sintomático que más de un tercio de los alumnos (35.1\%) haya tomado decisiones obligadas con base en criterios no académicos, relacionados con deficiencias en dichos procesos: $13.3 \%$ se obligó por la necesidad de cubrir créditos; $12.9 \%$ por la compatibilidad de horarios, cuya administración ha sido problemática para la Unidad, dada la insuficiencia de aulas; y 8.7\%, por la casualidad de que era donde aún había cupo a la hora de inscribirse. Sobre todo, las dos últimas respuestas indican problemas de improvisación y planificación al momento en que el alumno toma decisiones académicas. Decisiones forzadas, en parte, por las deficiencias en los procesos de toma de decisiones sobre la oferta de las OEX, la cual debería resultar de una participación colegiada multidisciplinar, como recomienda la REDIC (Quintá, 2014).

Esta información es importante a la luz de procesos en los que la contribución institucional parece débil como fuente de información y de orientación, porque permite bosquejar a una parte destacable de alumnos legítimamente preocupados por su formación: $14.3 \%$ manifestó estar interesado en la obtención de conocimientos teóricos o prácticos a través de las OEX; $13.9 \%$ en cuidar que éstas tuvieran alguna relación con la carrera; y $2.8 \%$, en el contenido de la asignatura, lo cual supone una revisión del programa de la OEX (cuadro 7). 
Cuadro 7. Aspectos por los cuales fueron elegidas las multidisciplinarias

\begin{tabular}{|c|c|c|c|}
\hline Razones & Frec. & $\%$ & Tipo de razones \\
\hline El nombre me pareció interesante & 150 & 32.7 & \multirow{6}{*}{$\begin{array}{c}\text { Académicas } \\
298 \text { alumnos } \\
64.9 \%\end{array}$} \\
\hline Consideré que estaba orientada a mi carrera & 64 & 13.9 & \\
\hline Para obtener conocimientos teóricos & 36 & 7.8 & \\
\hline PARA GANAR CONOCIMIENTOS PRÁCTICOS & 30 & 6.5 & \\
\hline Interés por el contenido & 13 & 2.8 & \\
\hline Otra razón académica & 5 & 1.1 & \\
\hline Necesitaba cubrir créditos & 61 & 13.3 & \multirow{4}{*}{$\begin{array}{c}\text { No académicas } \\
161 \text { alumnos } \\
35.1 \%\end{array}$} \\
\hline POR EL HORARIO & 59 & 12.9 & \\
\hline Fue donde había cupo & 40 & 8.7 & \\
\hline Otra razón no académica & 1 & 0.2 & \\
\hline Total & 459 & 100 & \\
\hline
\end{tabular}

Fuente: cálculos propios con base en el censo La multidisciplina desde la experiencia de los alumnos, UAM-I 2014.

Para proporciones importantes de los alumnos, el momento de la inscripción en OEX fue particularmente problemático, $77.3 \%$ expresó que encontrar cupo fue un problema y 44\% consideró que el horario no se adaptó a sus necesidades (cuadro 8).
Cuando los alumnos no pueden superar esas dificultades, terminan por inscribirse en la opción que tienen a la mano, sin importar la relevancia de la misma para su formación académica y dejando que imperen los referentes con intensidad pragmática.

\section{Cuadro 8. Dificultades al momento de la inscripción}

\begin{tabular}{l|c|c|c}
\multicolumn{1}{c|}{ Tipo de dificultad } & Desacuerdo & Acuerdo & Total \\
\hline El cupo fue un problema & 22.7 & 77.3 & 100 \\
\hline El horario no se adaptó a mis necesidades & 56.0 & 44.0 & 100 \\
\hline
\end{tabular}

Fuente: cálculos propios con base en el censo La multidisciplina desde la experiencia de los alumnos, UAM-I 2014. 
No aparecieron mayores evidencias de que los alumnos puedan diferenciar partes del proceso constitutivo de las OEX. No logran diferenciar entre el diseño y la operación de la estrategia institucional de flexibilidad curricular. Tienen escasa información para comprender el significado académico de cursar las OEX, lo cual propicia que centren su atención en las cuestiones administrativas. Esta complicada situación, experimentada por los alumnos, se ha agudizado y ya parece crónica, porque en general tampoco expresan capacidades de autonomía, ni voluntad de informarse acerca de las OEX que han de cursar. Se vive una realidad en la que los actores no asumen su parte de corresponsabilidad en los procesos de enseñanza-aprendizaje.

Se puede suponer la existencia de una racionalidad instrumental imperante en la elección de OEX que influye en el desempeño de los alumnos en esas asignaturas, pero carecemos de información sobre esa relación; sin embargo, podemos tener una aproximación sobre las expectativas que tenían los alumnos, y qué tanto éstas se cumplieron al cursarlas.

Pese a que el alumnado accede a escasa y poco adecuada información y orientación académica sobre las OEX a cursar, más de tres cuartas partes $(77.1 \%)$ manifestó opiniones favorables sobre el cumplimiento de sus expectativas (suponemos que éstas pueden estar más referidas a lo administrativo que a lo académico) y el resto, cercano a la cuarta parte del alumnado, apreció que esta clase de asignaturas poco o nada había cubierto sus intereses personales (22.9\%) (cuadro 9).

Un hallazgo destacable es la existencia de evidencia estadística (Valor $\mathrm{P}=0.000$ ) para sostener que los alumnos cuya elección descansó en razones no académicas, tendieron a manifestar insatisfacción con las OEX elegidas, en comparación con quienes esgrimieron razones académicas ( $\mathrm{V}$ de Cramer= 0.2294). Entre los primeros, $36 \%$ reportaron que poco o nada se habían cubierto sus expectativas, mientras que sólo $15.8 \%$ de los segundos emitieron afirmaciones en el mismo sentido (cuadro 9).

Las percepciones de los alumnos sobre el conjunto de las OEX, mismas que aparecen como fundamentales en los planes de estudio, exigen ser consideradas para una revitalización de la interdisciplinariedad en la UAM-I, ya que suponemos que las decisiones, en buena medida utilitarias, de los alumnos en la elección de esas asignaturas, estén del todo expresando logros de esa estrategia institucional, más aún cuando la propia institución no ha hecho seguimiento evaluando sus resultados. Buena parte de dichas percepciones dibujan una situación preocupante de la gestión académica, que suele propiciar quejas de los alumnos sobre la cuestión administrativa referida a la oferta y los cupos, que al final termina condicionando las necesidades formativas.

\section{Cuadro 9. Tipo de razón para elegir multidisciplinarias vs. cumplimiento de expectativas (\%)}

\begin{tabular}{|c|c|c|c|}
\hline \multirow{2}{*}{ Tipo de razones } & \multicolumn{3}{|c|}{ ¿Qué tanto se cumplieron tus expectativas? } \\
\hline & Poco o nada & Algo o totalmente & Total \\
\hline No académicas & 36.0 & 64.0 & 100 \\
\hline Académicas & 15.8 & 84.2 & 100 \\
\hline Total & 22.9 & 77.1 & 100 \\
\hline \multicolumn{4}{|c|}{ Pearson Chi ${ }^{2}=24.1092 \operatorname{Pr}=0.000$ Cramér's V $=0.2294$} \\
\hline
\end{tabular}

Fuente: cálculos propios con base en el censo La multidisciplina desde la experiencia de los alumnos, UAM-I 2014. 


\section{Pertinencia y aceptación crítica}

En las PODI se especifica que la formación de los alumnos debe ser integral (UAM-I, 2003). Para atender esta disposición académica se procura que los alumnos, a través de las OEX, por un lado, fortalezcan sus conocimientos disciplinares, exponiéndolos a saberes de otras formas científicas de conocer la realidad y, por otro lado, paralelamente se aproximen a contribuciones culturales que amplíen sus horizontes cognitivos. La idea central de esta estrategia institucional radica en la expectativa de que el alumnado, al final de sus estudios universitarios, haya ampliado sus oportunidades tanto de desarrollo personal y profesional, como de participación en los mercados laborales. Eso, entonces, desde la perspectiva institucional parece lo más acotado de la pertinencia educativa dispuesta. A ese respecto se encontró que los alumnos perciben diferenciadamente el logro de estas aspiraciones y que, pese a las dificultades enfrentadas para cursar algunas OEX, consideran que estas cumplen con sus expectativas.

Respecto a la pertinencia de las OEX, más de la mitad de los consultados $(57.3 \%)$ no quiere que haya más de estas asignaturas en los planes de estudio (cuadro 10), pero también piensa que en sí pueden servir para un mejor ejercicio profesional $(60 \%)$, por lo que no deberían de eliminarse $(58.9 \%)$ y no está de acuerdo con la afirmación de que no sirven de mucho $(56.7 \%)$. Por lo mismo, lo que debería de hacerse es mejorarlas, según la enorme mayoría (82.3\%).

\section{Cuadro 10. Preguntas sobre pertinencia de las OEX}

\begin{tabular}{|c|c|c|c|}
\hline Pregunta & Desacuerdo & Acuerdo & Total \\
\hline Se han cubierto las expectativas por las cuales elegí la extradivisional que estoy cursando & 22.9 & 77.1 & 100 \\
\hline La extradivisional es acorde con la orientación general de mi carrera & 52.1 & 47.9 & 100 \\
\hline Me está dando conocimientos teóricos útiles para mi vida laboral & 47.8 & 52.2 & 100 \\
\hline Me está dando conocimientos prácticos útiles para mi vida laboral & 53.7 & 46.3 & 100 \\
\hline Me está dando conocimientos generales para mi desarrollo como persona & 15.7 & 84.2 & 100 \\
\hline Tengo suficientes conocimientos previos para entender los contenidos de la extradivisional & 37.7 & 62.3 & 100 \\
\hline Es importante tener extradivisionales para mejorar el ejercicio de la profesión & 40.0 & 60.0 & 100 \\
\hline $\begin{array}{l}\text { Las extradivisionales sirven para adquirir conocimientos que me serán de utilidad a la hora de buscar } \\
\text { trabajo }\end{array}$ & 54.7 & 45.3 & 100 \\
\hline Debería de haber más extradivisionales & 57.3 & 42.7 & 100 \\
\hline Las extradivisionales deberían de eliminarse & 58.9 & 41.0 & 100 \\
\hline Las extradivisionales $\mathrm{NO}$ sirven de mucho & 56.7 & 43.4 & 100 \\
\hline Debería mejorarse el diseño de las extradivisionales & 17.7 & 82.3 & 100 \\
\hline \multicolumn{4}{|l|}{ Información complementaria } \\
\hline LA EXTRADIVISIONAL ME DA INSOMNIO & 75.8 & 24.2 & 100 \\
\hline Al inscribirme, el horario de la extradivisional se adaptó a mis necesidades & 44.0 & 56.0 & 100 \\
\hline
\end{tabular}

Fuente: cálculos propios con base en el censo La multidisciplina desde la experiencia de los alumnos, UAM-1 2014. 
Estas percepciones indican la existencia de elevada aceptación, en combinación con fuerte cuestionamiento hacia las OEX, porque es evidente el desacuerdo hacia la situación actual de las mismas, el cual no debe desestimarse, como sugieren varios especialistas (Gil, 2012 y Popkewitz, 2000). Tal desacuerdo, desde la perspectiva de los alumnos, se basa en diferentes fundamentos académicos y prácticos, que ponen en duda los aportes de las asignaturas actuales, en términos de su pertinencia.

Como ya se mencionó antes, en las percepciones positivas de los alumnos tiene un fuerte anclaje la apreciación de que las OEX han cumplido con las expectativas que tenían cuando las eligieron $(77.1 \%)$ (cuadro 10). Esta valoración encuentra asidero sobre todo en la apreciación mayoritaria de que las OEX contribuyen al desarrollo personal (84.2\%). No obstante, es preocupante que más de la mitad de los alumnos no les encuentren relación con su formación disciplinar (52.1\%). Vale insistir en que esta clase de valoraciones encuentran sustento en las cuestiones operativas, más que en las académicas, ya que hay alumnos que terminan aceptando lo que se les ofrece, como en la carrera de Sociología, donde frecuentemente terminan cursando optativas como Química Orgánica, que en principio no tiene congruencia académica específica.

En el mismo sentido, también preocupan las expresiones de incertidumbre sobre el impacto académico de las OEX en las posibilidades de inserción exitosa a los mercados de trabajo. Más de la mitad, o cerca de esa proporción, consideran que no sirven de mucho para obtener conocimientos que faciliten la entrada a los mercados de trabajo (54.7\%), o dudan que los conocimientos prácticos $(53.7 \%)$ y teóricos ofrecidos $(47.8 \%)$ tengan utilidad en su vida laboral (cuadro 11). Esto devela sus dudas acerca de la adquisición de habilidades y herramientas adecuadas para enfrentarse a un entorno laboral cada vez más exigente y competitivo, amén de discriminatorio y excluyente respecto a los egresados de universidades públicas.
Otro hallazgo de esta investigación está en la clase de valoraciones que los estudiantes tienen sobre la suficiencia de sus conocimientos previamente adquiridos para entender con solvencia los contenidos que se ofrecen en las OEX. En este sentido, cerca del $40 \%$ dijo no tener suficientes conocimientos previos. Esta es una cuestión importante en tanto que contradice el sentido de algunas opiniones vertidas por profesores asignados a esas asignaturas. Como parte de las valoraciones de pasillo que suelen ocurrir en el día a día, hay quienes sienten la necesidad de realizar esfuerzos extraordinarios para alinear, pragmáticamente, los contenidos al tipo de alumnado que han de atender. Otros sienten que no pueden hacer algo al respecto, y terminan ofreciendo "lo que se pueda" ante la impotencia de enfrentar el reto de construir ambientes de aprendizaje idóneos para poblaciones multidisciplinares.

La información empírica develó problemáticas notables en la gestión administrativa, que afectan la eficacia académica de las OEX. Los horarios en que se ofrecen estas asignaturas forjan percepciones divididas, aunque ligeramente más positivas que negativas. Entre los alumnos, 44\% manifestó que los horarios no se adaptaron a sus necesidades. Esta proporción, relativamente elevada, constituye en sí una fuerte crítica a la capacidad adaptativa de la gestión administrativa, ante la necesidad institucional de lanzar una oferta de formación universitaria inclusiva a grupos poblacionales que sufren carencias estructurales importantes, tanto en lo económico como en lo académico. A pesar de que pueda esgrimirse que en esta institución los alumnos de tiempo completo deben estar a disposición de la academia entre las 7:00 y las 20:00 horas, es un hecho probado que un tercio de los matriculados tienen que trabajar para enfrentar problemas cotidianos de bienestar mínimo (Hernández et al., 2017; Rodríguez y Hernández, 2008).

Evidentemente, preocupa notablemente a los alumnos la imposibilidad de encontrar cupo en los grupos que se ofrecen. De hecho, hay asignaturas 
cuya elevada demanda estudiantil provoca la saturación de los grupos, desde el momento mismo de su apertura formal.

A nivel de las divisiones encontramos diferencias importantes. Los alumnos de CBS son los que percibieron con mayor positividad la pertinencia de las OEX, los de CSH expresaron apreciaciones críticas, aunque algunos las rechazan y los de CBI, en general, mostraron una ruta intermedia entre las otras dos divisiones. Destacamos del conjunto de apreciaciones que para los alumnos de CBS las OEX en general tienen pertinencia para su formación académica, el $72.7 \%$ expresó que deberían rediseñarse, no obstante, el $36.2 \%$ percibió que deberían eliminarse, y el $24.1 \%$ percibieron que les produce problemas de insomnio (cuadro 11).

\section{Cuadro 11. Porcentaje de acuerdo con cada afirmación, según sexo y división. Variables sobre pertinencia de las multidisciplinarias}

\begin{tabular}{|c|c|c|c|c|c|c|}
\hline Fuente de orientación & $\boldsymbol{H}$ & M & CBI & CSH & CBS & Total \\
\hline $\begin{array}{l}\text { Se han cumplido las expectativas por las cuales elegí la extradivisional que } \\
\text { estoy cursando }\end{array}$ & 78.5 & 75.7 & 74.1 & 76.3 & 82.5 & 76.9 \\
\hline La extradivisional es acorde con la orientación general de mi carrera & 49.7 & 46.5 & 53.8 & 45.0 & 63.8 & 47.9 \\
\hline Me está dando conocimientos teóricos útiles para mi vida laboral & 53.0 & 51.5 & 46.2 & 50.5 & 65.5 & 52.2 \\
\hline Me está dando conocimientos prácticos útiles para mi vida laboral & 43.9 & 48.1 & 30.8 & 45.7 & 56.9 & 46.3 \\
\hline Me está dando conocimientos generales para mi desarrollo como persona & 85.9 & 83.0 & 92.3 & 83.4 & 86.2 & 84.2 \\
\hline $\begin{array}{l}\text { Tengo suficientes conocimientos previos para entender los contenidos de la } \\
\text { extradivisional }\end{array}$ & 64.0 & 61.0 & 88.5 & 58.6 & 74.1 & 62.3 \\
\hline $\begin{array}{l}\text { Es importante tener extradivisionales para mejorar el ejercicio de la } \\
\text { profesión }\end{array}$ & 62.6 & 57.9 & 73.1 & 56.6 & 75.9 & 60.0 \\
\hline $\begin{array}{l}\text { Las extradivisionales sirven para adquirir conocimientos que me serán de } \\
\text { utilidad a la hora de buscar trabajo }\end{array}$ & 45.5 & 45.2 & 57.7 & 41.3 & 65.5 & 45.3 \\
\hline Debería de haber más extradivisionales & 42.6 & 42.7 & 53.8 & 39.1 & 60.3 & 42.7 \\
\hline Las extradivisionales deberían de eliminarse & 35.9 & 45.0 & 23.1 & 43.0 & 36.2 & 41.0 \\
\hline Las extradivisionales NO sirven de mucho & 41.6 & 44.6 & 42.3 & 44.2 & 37.9 & 43.3 \\
\hline Debería mejorarse el diseño de las extradivisionales & 86.2 & 79.3 & 64.0 & 84.9 & 72.7 & 82.3 \\
\hline \multicolumn{7}{|c|}{ Información complementaria } \\
\hline La extradivisional me da insomnio & 21.7 & 26.2 & 7.7 & 25.4 & 24.1 & 24.2 \\
\hline Al inscribirme, el horario de la extradivisional se adaptó a mis necesidades & 58.6 & 54.1 & 38.5 & 58.3 & 49.1 & 56.0 \\
\hline
\end{tabular}

H: Hombre; M: Mujer; CBI: Ciencias Básicas e Ingeniería; CSH: Ciencias Sociales y Humanidades; CBS: Ciencias Biológicas y de la Salud.

Fuente: cálculos propios con base en el censo La multidisciplina desde la experiencia de los alumnos, UAM-I 2014. 
En los alumnos de CSH se encuentran las percepciones más críticas con relación a la pertinencia de esas asignaturas. De las tres divisiones, los alumnos de CSH son los que registraron el porcentaje más alto $(84.9 \%)$ que proponen que esas OEX deberían ser rediseñadas, cifra que se convierte en una mayor preocupación al ser vinculada con las percepciones de que deben ser eliminadas (43\%) y de que no sirven de mucho $(44.2 \%)$, es necesario tomar esto en cuenta con seriedad académica ya que también están impactando la salud de los alumnos, el 25.4\% expresó que estas apreciaciones críticas les provocan insomnio (cuadro 11).

Los alumnos de la división de CBI expresaron una disposición más concordante que discordante hacia las OEX, sus opiniones se encuentran entre las otras dos divisiones, sólo en dos aspectos sobresalen de las otras dos: 92.3\% considera que impactan en su desarrollo personal y el $88.5 \%$ perciben que cuentan con los conocimientos previos para cursarlas (cuadro 11).

Es posible una aproximación a la pertinencia de las asignaturas OEX por medio del índice sintético PERT. Para el conjunto de la UAM-I, los valores adoptados por la media (30.8) y la mediana (31) de este índice prácticamente coinciden con el valor a la mitad de la escala (30), lo cual da idea de que las autoridades de la UAM-I necesitan poner más atención en el cumplimiento del marco normativo orientador de la docencia, en su momento presentado como novedoso, ya que prácticamente los valores adoptados por la media y la mediana de este índice de 30.8 y 31 coinciden con el valor a la mitad de la escala (30) (cuadro 12).

\section{Cuadro 12. Media y mediana del índice PERT}

\begin{tabular}{|c|c|c|c|c|c|c|c|c|c|c|c|c|}
\hline \multirow{2}{*}{ Valor a mitad de escala PERT } & \multicolumn{6}{|c|}{ Media } & \multicolumn{6}{|c|}{ Mediana } \\
\hline & $\boldsymbol{H}$ & M & CBI & CSH & CBS & Total & $\boldsymbol{H}$ & $M$ & CBI & CSH & CBS & Total \\
\hline 30 & 31.0 & 30.6 & 34.0 & 30.1 & 34.0 & 30.8 & 31.0 & 31.0 & 35.0 & 30.0 & 35.0 & 31.0 \\
\hline
\end{tabular}

PERT: Pertinencia de las Multidisciplinarias desde la perspectiva de los alumnos; H: Hombre; M: Mujer; CBI: Ciencias Básicas e Ingeniería; CSH: Ciencias Sociales y Humanidades; CBS: Ciencias Biológicas y de la Salud.

Fuente: cálculos propios con base en el censo La multidisciplina desde la experiencia de los alumnos, UAM-I 2014.

\section{Propuesta estudiantil}

La mejora del diseño curricular y los procesos administrativos relacionados con la oferta de asignaturas multidisciplinarias, requiere aportes de los estudiantes, basados en su experiencia escolar, no sólo relacionada con la docencia en general (Rocha, 2013 y Carranza et al., 2009), sino también con el haber cursado específicamente dicha clase de asignaturas. En este sentido, las opiniones de los alumnos muestran aceptación de las OEX, sin dejar de ser críticos sobre el diseño y las formas operativas de aplicación de esas asignaturas. Prevalece un sentido más inclinado hacia la valoración positiva que hacia la negativa, incidiendo en la constitución de propuestas con proclividad a mejorarlas de distintas maneras $(91.4 \%)$ que a suprimirlas o reducir su número $(8.6 \%$ ) (cuadro 13). Los alumnos propusieron 292 iniciativas que reclaman mejoramiento académico y transformación administrativa. 


\section{Cuadro 13. Propuestas de los alumnos para mejorar las asignaturas multidisciplinarias}

\begin{tabular}{l|c|c}
\multicolumn{1}{c|}{ Propuesta } & Frec. & (\%) \\
\hline Orientar los contenidos a la carrera o la división & 123 & 42.1 \\
\hline Ampliar cupo, abrir más grupos o diversificar horarios & 68 & 23.3 \\
\hline Eliminarlas o reducir su número & 25 & 8.6 \\
\hline Diversificar la oferta de interdisciplinarias & 24 & 8.2 \\
\hline Modificar la forma de enseñanza & 16 & 5.5 \\
\hline Que sean prácticas & 8 & 2.7 \\
\hline Fomentar o informar sobre las interdisciplinarias & 8 & 2.7 \\
\hline Otras (que no sean obligatorias, contenidos de desarrollo personal o artístico, ampliar \\
espacio en aulas, que no estén seriadas entre sí, etcétera)
\end{tabular}

Fuente: cálculos propios con base en el censo La multidisciplina desde la experiencia de los alumnos, UAM-I 2014.

Como se ha visto a lo largo de este documento, los alumnos expresaron preocupación por la incipiente vinculación de los conocimientos disciplinares propios de la licenciatura, con los propuestos en las OEX, cuestión que vuelve a saltar en las propuestas para su renovación, puesto que $42.1 \%$ de los alumnos insiste en orientar esos contenidos académicos a las necesidades disciplinares de la licenciatura que cursan o a la división a la que pertenecen. En menor medida, el 32.8\% de los alumnos sugirió la ampliación de cupos, incremento de grupos o diversificación de horarios (cuadro 13).

\section{Reflexión final}

Las innovaciones curriculares no pueden reducirse a simples recetas que se pueden aplicar en cualquier espacio educativo sin considerar a las comunidades universitarias que las van a impulsar, aplicar y padecer. Un cambio educativo de intensidad en la institución analizada tendría que registrarse a partir de las transformaciones de las prácticas docentes. Los planes de estudio y los programas de las asignaturas son, como dice Díaz Barriga (2010), "un foco intelectual y organizativo de los procesos educativos en las escuelas e instituciones educativas, el terreno donde se definen y debaten fines, contenidos y procesos; ante todo, es el espacio donde grupos y actores se disputan el poder en las instituciones".

Los alumnos ocupan un lugar especial en los discursos de reforma educativa y aun así se mantienen en la práctica como individuos consumidores de políticas, tales como la flexibilidad curricular que genera simpatías en ellos y a su vez le encuentran poco sentido para su formación disciplinar y acceso al mercado de trabajo, lo que indica que carecen de un entendimiento de los fines que persigue esa innovación curricular, reducida en la percepción de los alumnos a la obtención de créditos que estipula la institución para la titulación. Esta falta de sentido e identidad hacia la interdisciplinariedad es producto 
de las formas de comunicación y apoyos de la institución para su comprensión, que persisten en recurrentes prácticas informativas y de orientación académica, como las tutorías, que ya han demostrado ser ineficientes y pese a ello se mantienen sin la menor consideración de los impactos negativos que tienen en los alumnos.

Lo expuesto evidencia que la flexibilidad curricular, como ha sido conceptualizada, poco se parece con lo que sucede en su aplicación, además de generar falta de sentido en la formación académica de los alumnos; tampoco da suficiente soporte práctico y administrativo para que la elección de las OEX tenga un carácter académico. Llama la atención que la posición de los alumnos sea favorable a una formación interdisciplinaria, pero rechazan una estrategia que se presenta en su experiencia como un desorden académico y que los limita en su formación, además de quitar espacio, en el curriculum escolar, a lo que ellos consideran como primordial: la adquisición de conocimiento disciplinar.

Los alumnos en su mayoría manifestaron su acuerdo hacia la interdisciplinariedad siempre y cuando sea reactualizada, lo interesante de su posicionamiento es el caudal de propuestas que realizan en el sentido de enriquecerla para que sea eficiente en su formación académica, lo que nos permite establecer que pueden ser considerados en el proceso de recambio, desde el momento que tienen conocimiento, experiencia y conciencia de un acto educativo que ellos sobrellevan y sin embargo, en lugar de proponer su eliminación expresan potenciales de apropiación desde un horizonte abierto a esa innovación curricular.

Por último hay que señalar que lo que hemos encontrado se suma a los escasos estudios realizados para entender el comportamiento de los alumnos, esperando identificar una serie de retos y oportunidades que permitan motivar a la investigación educativa para la creación de innovaciones curriculares que rebasen el momento de la moda, de las presiones a que son sujetas para acceder a la órbita de la mercantilización de la educación y al cumplimiento de prescripciones en torno a los financiamientos que puede obtener, y poder construir una nueva senda en la que los actores educativos involucrados sean considerados como sujetos y no meros objetos de aplicación de políticas educativas. 


\section{Referencias}

Caamal, Febe y Pedro Canto (2009), "Flexibilidad curricular: opinión de estudiantes y docentes universitarios", ponencia presentada en el $X$ Congreso Nacional de Investigación Educativa, COMIE, Veracruz, México.

Cano, Rufino (2009), "Tutoría universitaria y aprendizaje por competencias. ¿Cómo lograrlo?”, Revista Electrónica Interuniversitaria de Formación del Profesorado, vol. 12 núm. 1, pp. 181-204, <https://dialnet.unirioja.es/descarga/ articulo/2956810.pdf> [Consulta: agosto de 2018].

Carranza Peña, María Guadalupe, Armando Ruiz Badillo y Jessica Cervantes Ramírez (2009), "Dos miradas sobre la misma práctica. Las concepciones de profesores y estudiantes en la UPN", X Congreso de Investigación Educativa, COMIE, Veracruz, México.

Consejo Europeo (2002), Conclusiones de la presidenciaBarcelona 15 y 16 de marzo de 2002, <https://www. consilium.europa.eu/media/20933/70829.pdf> [Consulta: julio de 2018].

De la Peña, Luis (2007), "La interdisciplina como meta", en Julio Muñoz (coord.), La interdisciplina y las grandes teorias del mundo moderno, México, UNAM/CIICYH, pp. 253-278.

Díaz Barriga, Ángel (2005), "El profesor de educación superior frente a las demandas de los nuevos debates educativos", Perfiles Educativos, vol. 27, núm. 108, pp. 9-30.

Díaz Barriga, Frida (2010), "Los profesores ante las innovaciones curriculares", en Revista Iberoamericana de Educación Superior (RIES), México, IISUE-UNAM/ Universia, vol. 1, núm. 1, pp. 37-57, <https://www. ries.universia.unam. $\mathrm{mx} / \mathrm{index} . \mathrm{php} / \mathrm{ries} / \mathrm{article} /$ view/15/56> [Consulta: junio de 2018].

Díaz Barriga, Frida (2005), "Desarrollo del currículo e innovación: modelos e investigación en los noventa", Perfiles Educativos, vol. 27, núm. 107, pp. 57-84, <http:// www.scielo.org.mx/scielo.php? script $=$ sci_arttext $\& p$ $\mathrm{id}=\mathrm{S} 018526982005000300004 \& \operatorname{lng}=\mathrm{es} \& \ln \mathrm{ln}=\mathrm{es}>$ [Consulta: febrero de 2019].
Escudero, Juan Manuel (2006), El espacio europeo de educación superior ¿ iSerá la hora de la renovación pedagógica de la universidad?, Murcia, ICE/Universidad de Murcia.

Follari, Roberto (2007), "La interdisciplina en la docencia", Polis, Revista de la Universidad Bolivariana, vol. 6, núm. 16, pp. 1-12, <https://www.redalyc.org/articulo. oa?id=30501603> [Consulta: febrero de 2018].

García, Teresa (2010), "La mercantilización de la educación", Revista Electrónica Interuniversitaria de Formación del Profesorado, vol. 13, núm. 2, pp. 16-21, <https://www.redalyc.org/pdf/2170/217014950002. pdf $>$ [Consulta: febrero de 2018].

Gil, Manuel (2012), "Hacia una reforma educativa desde la perspectiva de las aulas", Perfiles Educativos, vol. 34, núm. especial, pp. 163.

Hargreaves, Andy y Dean Fink (2006), "Estrategias de cambio y mejora en educación caracterizadas por su relevancia, difusión y continuidad en el tiempo", Revista de Educación, núm. 339, pp. 43-58.

Hernández, Juan Manuel y Marco Leyva (2015), "La interdisciplina en la UAM-Iztapalapa desde la experiencia de los alumnos 2014" (Documento no publicado), México, UAM-Iztapalapa.

Hernández, Juan Manuel y Javier Rodríguez (2015), "La reforma educativa en la UAM-Iztapalapa. La formación profesional y el campo laboral desde la perspectiva de los estudiantes", reporte de investigación (Documento no publicado), México, Universidad Autónoma Metropolitana.

Hernández, Juan Manuel, Javier Rodríguez y Marco Leyva (2017), “CCómo es el ingreso a la licenciatura en Sociología de la UAM-I?", ponencia presentada en las fornadas de Sociología 2017. Pensar sociológicamente, México, UAM-Iztapalapa, 4-6 de octubre.

Kravzov, Esther(2000), "Una experienciainterdisciplinaria”, ponencia presentada en el Primer Encuentro. La Experiencia Interdisciplinaria en la Universidad, México, UNAM-Centro de Investigaciones Interdisciplinarias en Ciencias y Humanidades, 27-30 de noviembre. 
Maldonado, Alma(2000), "Los organismos internacionales y la educación en México: El caso de la educación superior y el Banco Mundial", Perfiles Educativos, vol. 22, núm. 87, pp. 51-75, <http://www.scielo.org.mx/scielo.php?script =sci_arttext\&pid=S0185-26982000000100004> [Consulta: noviembre de 2018].

Martínez, Lilia, David Toledo y Román Rey (2009), "El currículo frente al pensamiento de la innovación. Un estudio en algunas carreras de ingeniería”, ponencia presentada en el $X$ Congreso Nacional de Investigación Educativa, COMIE, Veracruz, México.

Ministros Europeos de Educación Superior (2003), "Berlín 2003. Educación Superior Europea”, España, Espacio Europeo de Educación Superior, <http://www.eees. es/pdf/Berlin_ES.pdf> [Consulta: julio de 2018].

Ministros Europeos de Educación Superior (1999), "The Bologna Declaration of 19 June 1999", España, Espacio Europeo de Educación Superior, <http://www.eees. es/pdf/Declaracion_Bolonia.pdf $>$ [Consulta: julio de 2018].

Ministros Europeos de Educación Superior (1998), "Declaración de La Sorbona", 25 de mayo, París, <https:/ / www.madrimasd.org/uploads/1998_ sorbonne_declaration_spanish_552622.pdf> [Consulta: julio de 2018].

Montes Silva, Melanie Elizabeth y Guadalupe López (2017), "Literacidad y alfabetización disciplinar: enfoques teóricos y propuestas pedagógicas", Perfiles Educativos, vol. 39, núm. 155, pp. 162-178, <http:// www.scielo.org.mx/scielo.php?script $=$ sci_arttext $\& p$ $\mathrm{id}=$ S0 $18526982017000100162 \& \operatorname{lng}=\mathrm{es} \& \operatorname{tng}=\mathrm{es}>$ [Consulta: febrero de 2019].

Morín, Edgar(1998), "Sobre la reforma de la Universidad", en Jaume Porte y Manuel Lladonosa (coords.), La Universidad en el cambio de siglo, Madrid, Alianza, pp. 19-28.

Popkewitz, Thomas (2000), Sociología política de las reformas educativas, Madrid, Ediciones Morata.

Quintá, María Cristina (2014), "Estrategias docentes para la formación interdisciplinar en educación superior", en Red Innova Cesal, Estrategias docentes para la formación interdisciplinar en educación superior, México,
REDIC, pp. 5-29.

Rocha, Reynaldo (2013), "Escala de opinión de los estudiantes sobre la efectividad de la docencia (EOEED) en educación superior", Revista Formación Universitaria, vol. 6, pp. 13-22.

Rodríguez, Javier y Juan Manuel Hernández (2008), "La deserción escolar universitaria en México. La experiencia de la Universidad Autónoma Metropolitana", Revista Electrónica Actualidades Investigativas en Educación, vol. 8, núm. 1, pp. 1-30.

Salvador, José Loreto (2014), "Transdisciplinariedad, complejidad y educación”, en María del Rosario Guerra (coord.), Debates éticos con metodología transdisciplinaria, México, Plaza y Valdés, pp. 61-87.

Santoyo, S. R. (1996), "Recomendaciones de la ANUIES en el diseño curricular", en ANUIES, Memorias del Encuentro de Modelos Curriculares de las Instituciones de Educación Superior, México, ANUIES-Región CentroSur, pp. 7-16.

SEP (2016), "El Modelo Educativo en México: el planteamiento pedagógico de la Reforma Educativa", Perfiles Educativos, vol. 38, núm. 154, pp. 216-225, <http://www.scielo.org.mx/scielo.php?script=sci_art text\&pid=S018526982016000400012\&lng=es\&tlng= es> [Consulta: diciembre de 2018].

Suárez, Daniel (2011), "Relatos de experiencia, saber pedagógico y reconstrucción de la memoria escolar", Educação em Revista, vol. 27, núm. 1, pp. 387-416, DOI: https://dx.doi.org/10.1590/S010246982011000100018.

Spelt, Elisabeth, Harm Biemans, Hidle Tobi, Pieternel Luning y Martin Mulder (2009), "Teaching and learning in interdisciplinary higher education: a systematic review", Educational Psychology Review, vol. 21, núm. 4, pp. 365-378.

Torres, Jurjo (1998), Globalización e interdisciplinariedad: el curriculo integrado, Madrid, Ediciones Morata.

UAM (2018), "Políticas operacionales de docencia", en Legislación Universitaria, México, UAM, pp. 255-258, <https://www.uam.mx/legislacion/pod/> [Consulta: diciembre de 2017]. 
UAM (2001), Una reflexión sobre la universidad desde la docencia, México, UAM.

UAM-I (2003), Políticas operativas de docencia de la Unidad Iztapalapa, <http://www2.izt.uam.mx/conacad/doc_ relevantes/politicas/pol_operativas_docencia.pdf $>$ [Consulta: febrero de 2018].

UNESCO (1998), "Declaración Mundial sobre la Educación Superior en el Siglo XXI: visión y acción", en Conferencia Mundial sobre la Educación Superior. Tomo 1. Informe final, 9 de octubre, pp. 19-30, París, <https:// unesdoc.unesco.org/ark:/48223/pf0000116345_spa>. Ziegler, Sandra (2003), "Los docentes como lectores de documentos curriculares. Aportes para el análisis de la recepción de la reforma curricular de los años noventa en Argentina", Revista Mexicana de Investigación Educativa, vol. 8, núm. 19, pp. 653-677.

\section{Cómo citar este artículo:}

Hernández-Vázquez, Juan-Manuel, Marco-Antonio Leyva-Piña y Javier Rodríguez-Laguna (2020), "La multidisciplina en los estudios universitarios. La perspectiva de los alumnos de la UAM-Iztapalapa", Revista Iberoamericana de Educación Superior (RIES), vol. XI, núm. 32, pp. 23-45, DOI: https://doi.org/10.22201/iisue.20072872e.2020.32.811 [Consulta: fecha de última consulta]. 